\title{
Resultados a largo plazo de pacientes con adenocarcinoma de recto inferior tratados con resección anterior ultrabaja interesfintérica
}

\author{
Francisco López-Kostner ${ }^{1}$, Carlos Miranda F. ${ }^{1}$, Alejandro Zárate C. ${ }^{1,2}$, \\ Claudio Wainstein G. ${ }^{1}$, Udo Kronberg ${ }^{1}$ y Alejandra Ponce F. ${ }^{1}$
}

Long-term results of patients with adenocarcinoma of the lower rectum treated with anterior intersphinteric ultra-low-resection

Introduction: Intersphinteric resection (ISR) allows preserve sphincter function in selected patients with rectal cancer (RC). Notwithstanding, it can produce alterations in defecation. Aim: To analyze the oncological and functional results after an ISR. Materials and Method: Observational, analytical, cross-sectional study, in the period 2007-2016. Inclusion criteria: Patients submitted to ISR by RC with curative intention. All the patients had a follow-up in 2017. Analysis of functional evaluation were performed by JorgeWexner, LARS and Kirwan scale. Statistical analysis: Descriptive statistics and Kaplan-Meier method. Results: Of 21 patients; Fourteen (67\%) were male, average age: 59 years. Tumor location: $4 \mathrm{~cm}(2-6 \mathrm{~cm})$ from anal verge. Eighteen $(85.7 \%)$ patients received neoadjuvant therapy. All distal and radial margins were negative. One patient $(4.8 \%)$ had distant metastases and there was no locoregional recurrence. With a median follow-up of 76.3 (9.8-126.8) months, the 5-year global and disease-free survival was: $100 \%$ and $95 \%$ (CI: 90.1-99.9\%), respectively. With a median follow-up of 90 months (21.7-124.2); Functional evaluation was performed on 15/21 patients. The Jorge-Wexner score had a median of 13 (4-17) points, the LARS scale of 34 points and in Kirwan scale, four patients $(26.7 \%)$ showed good function (Kirwan I-II). Conclusion: The oncological results of patients undergoing ISR are satisfactory, however, functional results should be taken into account when proposing this surgical procedure.

Key words: rectal cancer; intersphinteric resection; oncological results; functional results.

\section{Resumen}

Introducción: La resección anterior ultrabaja interesfintérica (RAUBIE), permite preservar la función esfinteriana en pacientes seleccionados con cáncer de recto (CR). No obstante, puede producir alteraciones en la función evacuatoria y esfinteriana. Objetivo: Analizar los resultados oncológicos y funcionales luego de una RAUBIE. Material y Método: Estudio observacional, analítico, transversal, durante el período 2007 a 2016. Criterios de inclusión: Pacientes sometidos a RAUBIE por CR con intención curativa. Todos los pacientes tuvieron un seguimiento el año 2017. Para la evaluación funcional se usó la escala de JorgeWexner, LARS y Kirwan. Análisis estadístico: Estadística descriptiva y método de Kaplan-Meier. Resultados: De 21 pacientes; catorce (67\%) fueron varones, edad promedio: 59 años. Ubicación tumoral: $4 \mathrm{~cm}$ (2-6 cm) del margen anal. Dieciocho $(85,7 \%)$ pacientes recibieron neoadyuvancia. Todos los márgenes quirúrgicos distales y radiales fueron negativos. Un paciente $(4,8 \%)$ tuvo metástasis a distancia y no hubo recurrencia locorregional. Con una mediana de seguimiento de 76,3 (9,8-126,8) meses, la sobrevida global y libre de enfermedad a 5 años fue de: 100\% y 95\% (IC: 90,1-99,9\%), respectivamente. Con una mediana de seguimiento de 90 meses (21,7-124,2); se realizó la evaluación funcional a 15/21 pacientes. El puntaje de Jorge-Wexner tuvo una mediana de 13 (4-17) puntos, la escala de LARS de 34 puntos y en la escala de Kirwan, cuatro pacientes (26,7\%) mostraron una buena función (Kirwan I-II). Conclusión: Si bien los resultados oncológicos de los pacientes sometidos a una RAUBIE son satisfactorios, se debería tomar en cuenta los resultados funcionales al momento de proponer esta alternativa quirúrgica.

Palabras clave: cáncer de recto; resección anterior ultrabaja interesfintérica; resultados oncológicos; resultados funcionales. 


\section{Introducción}

El cáncer de recto $(\mathrm{CR})$ localizado en los últimos $5 \mathrm{~cm}$ del margen anal es un desafío para los cirujanos colorrectales; ya que debe cumplir dos objetivos fundamentales: asegurar un buen resultado oncológico y permitir una buena calidad de vida. Esta última se ve fuertemente afectada cuando se generan secuelas funcionales en la esfera defecatoria, urológica y sexual producto del daño de estructuras musculares y neurológicas ${ }^{1,2}$.

La resección abdómino-perineal (RAP) de Miles ha sido el tratamiento quirúrgico estándar para el adenocarcinoma de recto de esta localización durante el siglo $\mathrm{XX}^{3}$; sin embargo, la pérdida definitiva de la función evacuatoria por vía anal conlleva a limitaciones psicológicas y sociales que comprometen fuertemente la calidad de vida ${ }^{4}$.

A mediados de los 90 Schiessel et al., proponen la resección anterior ultrabaja interesfintérica (RAU$\mathrm{BIE}$ ); que además de obtener resultados oncológicos aceptables, permite preservar la mayor parte del complejo esfinteriano en pacientes seleccionados con un cáncer en los últimos $5 \mathrm{~cm}$ del recto siendo una alternativa a la RAP ${ }^{5,6}$.

Desde que la RAUBIE se inició, múltiples estudios y revisiones sistemáticas muestran la seguridad oncológica de esta técnica con recurrencias locales a 5 años que van de 0 a $23 \%$; así como supervivencias globales y libres de enfermedad que van desde 79 a $97 \%$ y 69 a $86 \%$, respectivamente $e^{7,8}$.

Si bien es cierto la RAUBIE permite preservar la mayor parte del complejo esfinteriano, puede producir alteraciones en la función evacuatoria que va a depender del nivel de la sección distal del esfínter anal interno (parcial, subtotal, extendida o total) lo que se acompañará de un grado variable de incontinencia fecal sumado al síndrome de resección anterior baja (síndrome LARS) ${ }^{10}$.

Como toda técnica recientemente introducida, los resultados funcionales post-RAUBIE eran aceptables; ; sin embargo, en una revisión sistemática realizada por Martin et al., el $2012^{7}$ se advertían una diversidad de resultados en lo referente a la continencia. En estos últimos 5 años se siguen publicando resultados heterogéneos en las diferentes escalas utilizadas para evaluar la función intestinal posquirúrgica, que se altera aún más si va asociado a terapia neoadyuvante; la cual ya está demostrada como factor de riesgo independiente en el deterioro de la función intestinal ${ }^{11}$.

El objetivo del presente estudio es analizar los resultados oncológicos y funcionales a largo plazo luego de una RAUBIE en pacientes con adenocarcinoma del recto inferior.

\section{Material y Métodos}

Estudio observacional, analítico, transversal de pacientes con CR inferior diagnosticados en la Clínica Las Condes durante el período 2007 a 2016. Los criterios de ingreso fueron pacientes sometidos a una RAUBIE con intención curativa, por adenocarcinoma invasor del recto inferior.

De acuerdo al protocolo de estadificación, los pacientes fueron sometidos a una resonancia de pelvis junto a una tomografía computada de tórax y abdomen y en casos seleccionados a una endosonografía rectal. La distancia del borde inferior del tumor al margen anal fue medida con un rectoscopio rígido. Selección de pacientes: Pacientes en quienes no hubiese invasión tumoral muscular (elevadores del ano, esfinter anal externo), presencia de una función esfinteriana satisfactoria, una localización tumoral que no permitiese una anastomosis mecánica primaria (menos de $2 \mathrm{~cm}$ entre el borde inferior del tumor y el plano de los elevadores). En todos los pacientes se inició la cirugía por vía transanal lo cual permitió definir el límite distal de sección y luego de desvincular el recto del aparato esfinteriano, se procedió a realizar una resección anterior ultrabaja por acceso laparoscópico. La pieza operatoria fue extraída por vía transanal y la reconstrucción del tránsito se realizó a través de una anastomosis coloanal directa manual. En todos los casos se desfuncionalizó la anastomosis a través de una ileostomía en asa transitoria.

Para la evaluación oncológica se tomaron en consideración los datos clínicos y quirúrgicos a través de la ficha electrónica de cada paciente. Para el análisis de los eventos adversos intrahospitalarios se utilizó la clasificación de Clavien-Dindo. La neoadyuvancia fue de curso largo que consistió generalmente en:

Protocolo de radiación de 45 Gy dividida en 25 fracciones seguido de una dosis adicional de 5,4 Gy para el área tumoral, asociado a 5 fluorouracilo cuya dosis fue de $225 \mathrm{mg} / \mathrm{m}^{2} /$ día durante el tiempo de la radioterapia. La respuesta patológica posneoadyuvancia se determinó mediante la escala de Ryan. También se evaluó la recurrencia locorregional y el desarrollo de metástasis a distancia; así como, la supervivencia global y libre de enfermedad a 5 años. Todos los pacientes tuvieron un seguimiento hasta el 31 de julio de 2017 y todos los fallecimientos se constataron mediante certificados de defunción.

En el seguimiento se realizó la evaluación funcional en todos los pacientes con más de 18 meses posterior al cierre de ileostomía y se determinó 


\section{ARTÍCULO ORIGINAL}

el grado de incontinencia fecal (Escala de JorgeWexner ${ }^{12}$ ) mediante una escala que consiste en no incontinencia: 0-2, incontinencia leve: 3-8, incontinencia moderada: 9-14 e incontinencia severa: 15-20; así también se categorizó en pacientes con buena función cuando el puntaje de la escala era $\leq$ a 10 y con mala función cuando el puntaje era $\geq a$ $16^{13}$. Además, se analizó la presencia del síndrome de resección anterior baja (Escala de LARS) y se categorizaron a los pacientes en no LARS: 0-20, LARS menor: 21-29 y LARS mayor: $30-42^{14}$. Por último se aplicó la escala de evaluación funcional de Kirwan (I-V) que se utiliza mayormente en países como Japón ${ }^{15}$, a la vez que se les subdividió en pacientes con buena función a los pacientes con evaluación funcional Kirwan $\mathrm{I}^{-\mathrm{II}^{\mathbf{1 6}}}$.

A todos los pacientes que se les realizó la evaluación funcional se les realizó la siguiente pregunta: ¿Qué preferiría; permanecer en su estado actual o portar una ostomía como en los primeros meses luego de su operación?, para estimar la preferencia de estas dos situaciones y extrapolarlas al escenario luego de realizarse una RAUBIE versus una RAP.

Para el análisis estadístico se utilizó estadística descriptiva y para el análisis de sobrevida el método de Kaplan-Meier, en lo que respecta a la evaluación oncológica y estadística descriptiva; así como, analítica $\left(\chi^{2}\right)$ en lo referente a la evaluación funcional.

Tabla 1. Características clínico-patológicas de los pacientes

\begin{tabular}{|lc|}
\hline Variables & Total $\mathbf{n}=\mathbf{2 1}$ \\
Edad (años-promedio) & $59(38,3-85,8)$ \\
Género (varones) & $14(67 \%)$ \\
Neoadyuvancia & $18(85,7 \%)$ \\
Ubicación tumoral (desde margen anal-mediana) & $4 \mathrm{~cm}(2-6)$ \\
Estadio clínico preoperatorio & \\
I & $3(14,3 \%)$ \\
II & $2(9,5 \%)$ \\
III & $15(71,4 \%)$ \\
IV & $1(4,8 \%)$ \\
Estadio anatomopatológico posoperatorio & \\
0 & $2(9,5 \%)$ \\
I & $12(57,1 \%)$ \\
II & $2(9,5 \%)$ \\
III & $4(19,1 \%)$ \\
IV & $1(4,8 \%)$ \\
\hline
\end{tabular}

\section{Resultados}

En el presente estudio se incluyeron un total de 21 pacientes; catorce $(67 \%)$ fueron varones, con una edad promedio de 59 años $(38,3-85,8)$. La mediana de la ubicación tumoral fue a $4 \mathrm{~cm}(2-6 \mathrm{~cm})$ del margen anal. Dieciocho pacientes $(85,7 \%)$ recibieron neoadyuvancia. Dieciséis pacientes $(76,2 \%)$ fueron estadios clínicos avanzados (III y IV) preneoadyuvancia (Tabla 1).

De los dieciocho pacientes que recibieron neoadyuvancia; los resultados de la pieza operatoria mostraron respuesta patológica completa en 2 (11\%). Los márgenes quirúrgicos distales y radiales fueron negativos en todas las piezas operatorias. La mediana del margen de resección distal fue de $10 \mathrm{~mm}(4-25 \mathrm{~mm})$. Todos los pacientes $(100 \%)$ tuvieron un margen de resección radial $>1 \mathrm{~mm}$. La mediana del número de linfonodos analizados en la pieza operatoria fue de 11 (1-24).

Una o más complicaciones se observaron en 12 pacientes (57\%). De ellos 5 pacientes fueron Clavien-Dindo III y IV, de los cuales 3 (14\%) fueron reoperados. Las reoperaciones fueron por necrosis del colon descendido en 2 casos y obstrucción intestinal en 1. No hubo mortalidad posoperatoria y la mediana de la estancia hospitalaria fue de 6 días (Figura 1).

Con una mediana de seguimiento de 76,3 meses (9,8-126,8); un paciente $(4,8 \%)$ desarrolló metástasis a distancia (pulmón) y ningún paciente desarrolló recurrencia locorregional. La supervivencia global y libre de enfermedad a 5 años fue de: $100 \%$ y 95\% (IC: 90,1-99,9\%) (Figura 2), respectivamente. Un paciente falleció a los 63 meses por causa no relacionada a la enfermedad (neumonía aspirativa).

La mediana de seguimiento de la evaluación funcional fue de 90 meses $(21,7-124,2)$ y fue realizada en 15 pacientes (Figura 3).

El puntaje de la escala de Jorge-Wexner tuvo una mediana de 13 puntos (4-17). Un 26,7\% presentó una buena función (puntaje $\leq 10$ ) y un $20 \%$ una mala función (puntaje $\geq 16$ ). La escala de LARS tuvo una mediana de 34 puntos (puntaje $\geq 30$ : 13 pacientes $(86,7 \%))$. Ni la escala de Jorge-Wexner (Hombres: 12 Mujeres: $13 \mathrm{p}=0,705$ ) ni la de LARS (Hombres: 33 Mujeres: $38 \mathrm{p}=0,053$ ) presentaron diferencias de puntaje según género. Lo mismo ocurre con ambas escalas entre los pacientes mayores y menores a 60 años, Jorge Wexner $(\geq 60$ : $12 v s<60: 12 \mathrm{p}=0,92)$ y LARS $(\geq 60: 33 v s<60$ : $36 \mathrm{p}=0,34)$. Con respecto a la escala de Kirwan, un $26,7 \%$ mostró una buena función (Kirwan I-II) (Tabla 2). 


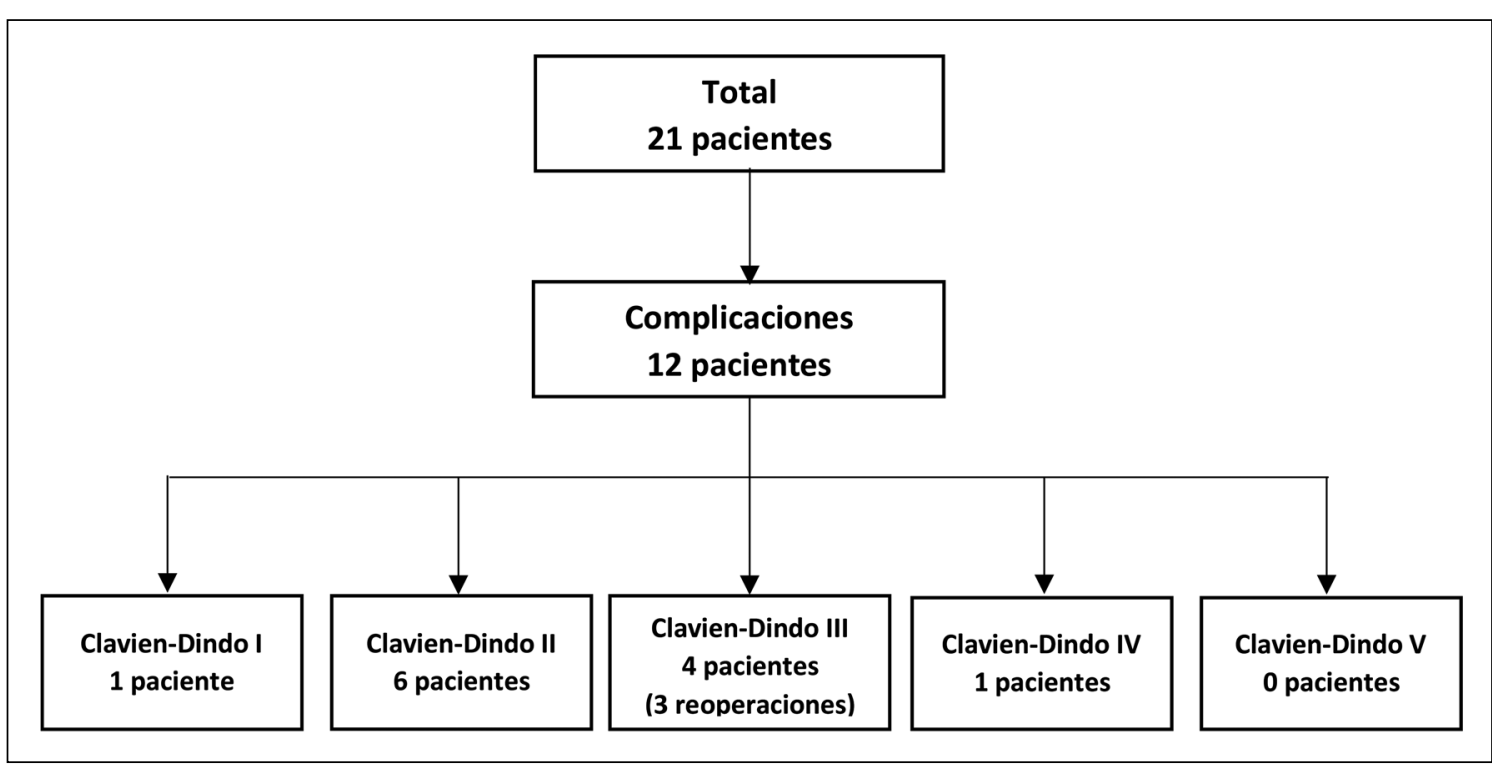

Figura 1. Clasificación Clavien-Dindo de los eventos adversos posquirúrgicos.

Con respecto a la pregunta sobre la preferencia de permanecer en su estado funcional actual o volver a una ostomía, el resultado mostró que trece (87\%) pacientes prefieren su estado actual a portar una ostomía.

\section{Discusión}

En esta serie se confirma la seguridad oncológica de esta técnica, ya que observamos ausencia de márgenes comprometidos en la pieza operatoria, ausencia de recurrencia local y elevada supervivencia tanto global, como la libre de enfermedad. Sin embargo, los resultados funcionales son variables y distan de los patrones de normalidad.

El iniciar la cirugía por vía transanal permite definir un margen distal y radial de entrada, lo que probablemente explique los buenos resultados observados en la pieza operatoria. Martin et al. ${ }^{7}$ y Akagi et al. ${ }^{8}$, describen márgenes negativos entre 92 al $100 \%$. En estos últimos 5 años se reportan publicaciones con tasas cercanas al 100\% ${ }^{6,13,17}$. El $100 \%$ de los pacientes de nuestra serie tuvieron un margen de resección radial $>1 \mathrm{~mm}$, Martin et al. ${ }^{7}$, describen un 96\% (89-100\%) y Akagi et al. ${ }^{8}$, reportan un rango entre $86,7-96 \%$; recientemente los reportes confirman valores cercanos al $100 \%{ }^{6,18}$. El margen de resección distal en nuestro estudio tuvo una mediana 10 (4-25) mm, Martín et al. ${ }^{7}$, informan un promedio de 17,1 (10-29) mm y Akagi et al. ${ }^{8}$, entre 5-20 $\mathrm{mm}$. Por otra parte en este trabajo la me-

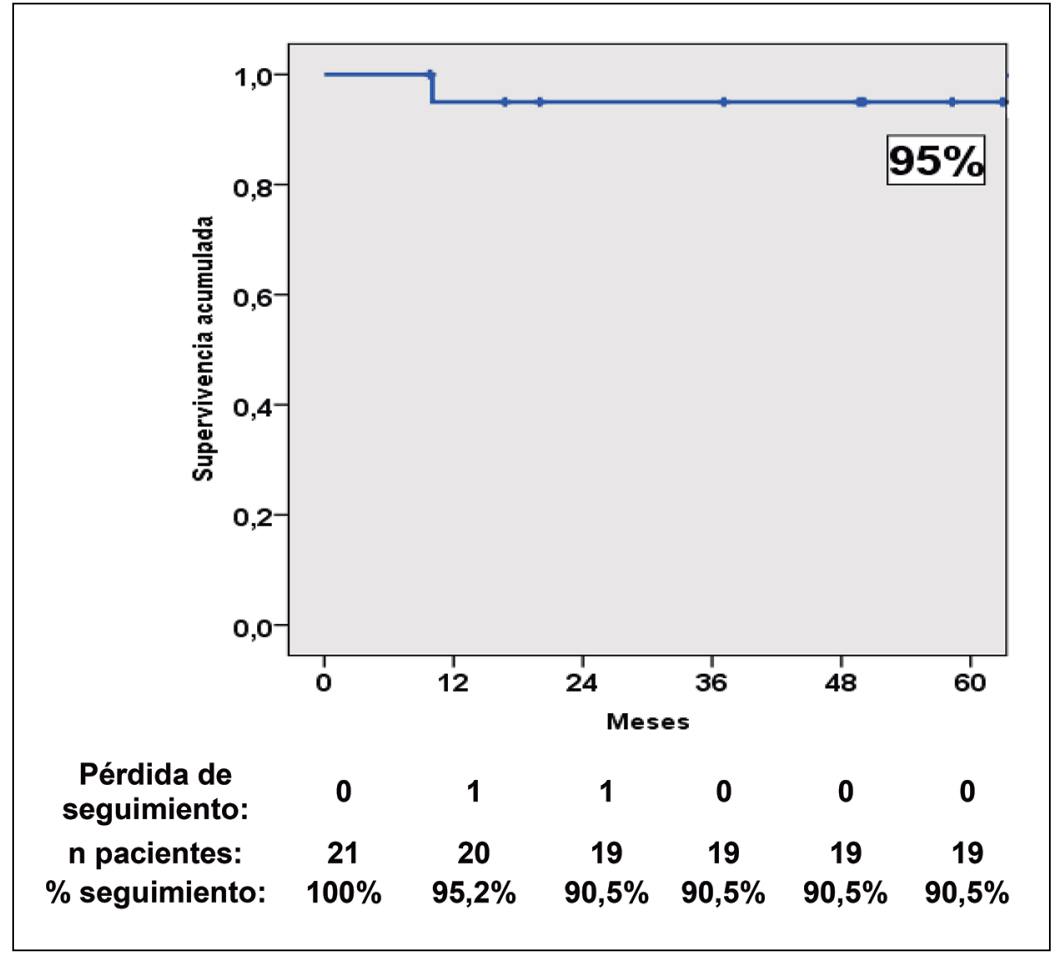

Figura 2. Supervivencia libre de enfermedad de los pacientes a 5 años.

diana del número de linfonodos resecados fue de 11 (1-24) que está dentro del rango (1-37) reportado en la literatura internacional ${ }^{16,19-22}$.

Respecto de la recurrencia local, se reporta un rango entre 0 y $23 \%$, con una media de $6 \%$ y 


\section{ARTÍ́CULO ORIGINAL}

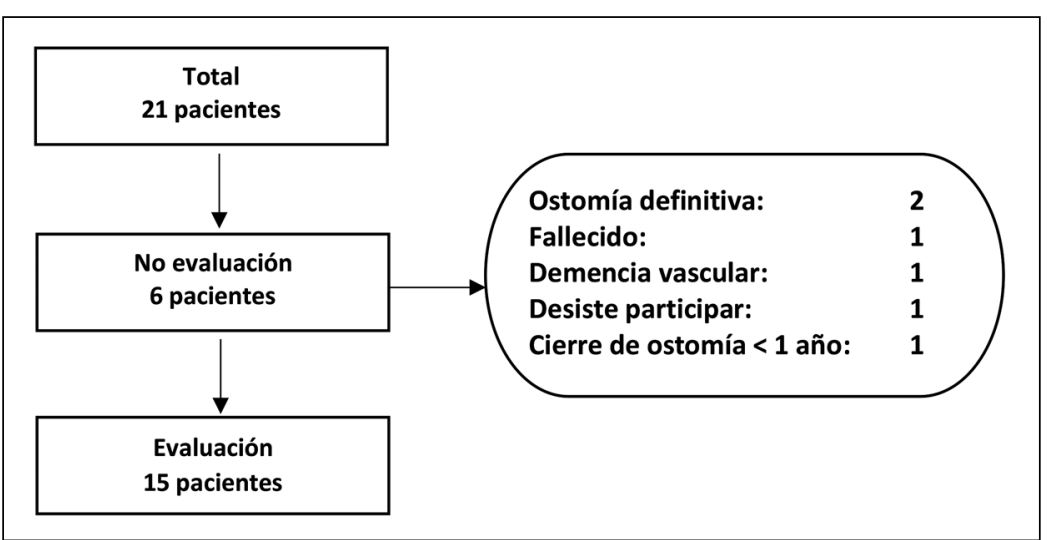

Figura 3. Flujograma de pacientes incluidos y excluidos en la evaluación funcional.

porcentajes de metástasis en un rango entre 2 a $19 \%{ }^{7,8}$. Con el advenimiento de la resección total del mesorrecto y la sinergia terapéutica de la neoadyuvancia al tratamiento quirúrgico, se pueden obtener recurrencias locales en un rango de $1,4 \%-3,2 \%{ }^{6,20,22}$.

Por otro lado, en los últimos 5 años, se han reportado resultados variables del desarrollo de metástasis en un intervalo de 5,1\%-21,6\% 6,13,17,18,20,23-26. Tsukamoto et al. ${ }^{17}$, describieron un $28,9 \%$ de estadios avanzados asociado a un $56,8 \%$ de tratamiento adyuvante, obteniendo un $11 \%$ de metástasis; lo opuesto a Koyama et $\mathrm{al}^{24}$, que con un $42 \%$ de estadios avanzados asociado a un $100 \%$ de terapia adyuvante, presentaron un $19,5 \%$ de metástasis.

La supervivencia global a 5 años de este estudio fue $100 \%$; Martín et al. ${ }^{7}$ presenta una media de $86 \%$ (62-97\%) y Akagi et al. ${ }^{8}$ un intervalo entre $79-97 \%$.

La supervivencia libre de enfermedad a 5 años en nuestra serie es de 95\%; Martin et al. ${ }^{7}$ muestran una media de 78,6\% (69-87\%) y Akagi et al. ${ }^{8}$ un intervalo entre $69-86 \%$, el primero explica el rango reportado de acuerdo a los resultados de $\mathrm{R}_{0}$ del $92 \%-100 \%$, un porcentaje de estadios avanzados y una falta de datos en el porcentaje de tratamiento neoadyuvante; originando un intervalo de recurrencia local entre el $0-23 \%$ y un intervalo de desarrollo de metástasis entre el 2,5\%-19\%. En los últimos 5 años esta supervivencia está sobre el $75 \% 0^{17,20,22,26,27}$.

Los resultados oncológicos favorables de este estudio, se pueden explicar en parte por el trabajo protocolizado de estudio preoperatorio, discusión ampliada en comité oncológico y experiencia del equipo quirúrgico.

Respecto a los resultados funcionales, en este estudio se observa una clara alteración de los valores en las diversas escalas, destacando una media de 13 puntos en la escala de Jorge y Wexner. En la litera-

Tabla 2. Resultados funcionales y probables factores asociados

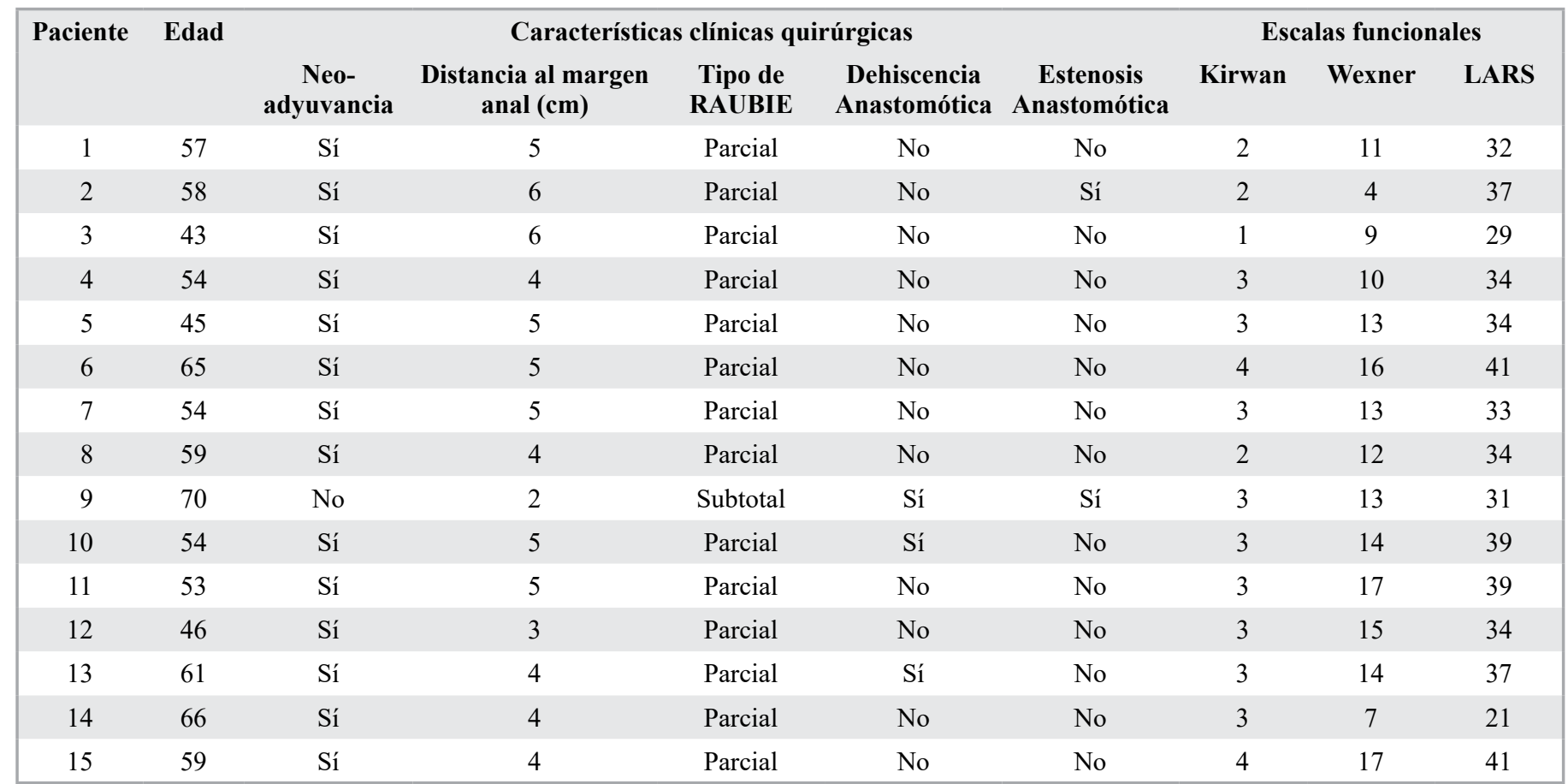


tura se presentan resultados ampliamente variables según escalas y factores quirúrgicos asociados.

Martin et al. $^{7}$ reportan un rango de continencia muy buena (escalas de Kirwan y Jorge-Wexner) entre $41-86,3 \%{ }^{19,28-30}$, siendo el tratamiento neoadyuvante menos del $50 \%$ en todos los estudios. Otros estudios reportan un valor de Wexner promedio de 15 (7-20) cuando todos los pacientes reciben neoadyuvancia ${ }^{13,31}$ y valores que van de 7,3-8,1 cuando la terapia neoadyuvante es menor al $15 \%{ }^{24,32}$. Saito et al. ${ }^{13}$, con un $100 \%$ de neoadyuvancia, observaron una disminución de 15 a 10 en el valor de Wexner a los 2 y 5 años luego del cierre de la ileostomía, respectivamente; este resultado difiere a lo obtenido en nuestra serie ya que el valor de Wexner se mantuvo constante. Actualmente, se ha adicionado una valoración a la escala de Wexner, denominándose pacientes con buena función a aquellos que tienen un valor de Wexner $\leq 10$ y pacientes con mala función a aquellos que tienen un valor de $\geq 16^{13}$. Los estudios mostraron rangos de buena función entre $60-83,3^{13,20,23,25}$ y de mala función de $19-20^{13,25}$ cuando la terapia neoadyuvante estuvo entre $0-33,9 \%$.

Respecto del LARS, Luca et al. ${ }^{16}$ con un 78,3\% de tratamiento neoadyuvante, reporta una porcentaje de LARS mayor de $23,8 \%$. Beppu et al. ${ }^{10}$ informa un $67,4 \%$ de LARS mayor cuando la terapia neoadyuvante se realizaba en el $100 \%$ de los casos. Otros estudios mostraron que a pesar que el tratamiento neoadyuvante estuvo en un rango de 62,5\%-100\%, tuvieron una buena función (Kirwan I-II) en un rango de $75,4 \%-91,7 \%{ }^{6,16,18}$.

Otros factores han sido también asociados con la alteración de la función intestinal ${ }^{10,13,33}$; como es el caso de las complicaciones posoperatorias, Yokota et al. ${ }^{25}$ analiza la repercusión en la función evacuatoria debido al desarrollo de la dehiscencia de anastomosis obteniendo una buena función de $20 \%$ y una mala función de $40 \%$, en la escala de Wexner. En pacientes que desarrollan estenosis crónica anastomótica obtuvieron un valor de Wexner de 13,6 \pm 3,9 en pacientes con estenosis en comparación a $9 \pm$ 4,5 en pacientes sin estenosis ${ }^{33}$.
Se ha descrito una tendencia a un mayor compromiso de la función intestinal cuando se realiza una RAUBIE subtotal o total en comparación a una RAUBIE parcial reflejado en un valor de Wexner de un 11,8 $\pm 2,6$ y 9,1 $\pm 5,6$; respectivamente ${ }^{33}$. En cambio, Saito et al. ${ }^{13}$ asocian al sexo masculino con un mayor compromiso de la función intestinal mostrando una mediana del valor de Wexner de 9 en comparación a un valor de 6,5 del sexo femenino $(p=0,046)$. En nuestro estudio no se encontraron asociaciones con los factores descritos.

Por lo mencionado, existen múltiples factores descritos que afectan la función intestinal y coexisten al momento de realizar una RAUBIE en el tratamiento del adenocarcinoma del recto inferior, explicando la amplia variabilidad de resultados que se han reportado hasta la actualidad.

Existieron algunas limitaciones en el presente estudio. Primero, los resultados se basan en una cohorte de pacientes retrospectiva realizada en una sola institución. Segundo, la población estudiada es pequeña impidiendo la evaluación de posibles factores de riesgo; que potencien a la neoadyuvancia, en la alteración de la función evacuatoria.

En conclusión, si bien los resultados oncológicos de los pacientes sometidos a una RAUBIE son satisfactorios, se debería tomar en cuenta los resultados funcionales al momento de proponer esta alternativa quirúrgica.

\section{Responsabilidades éticas}

Protección de personas y animales. Los autores declaran que para esta investigación no se han realizado experimentos en seres humanos ni en animales.

Confidencialidad de los datos. Los autores declaran que en este artículo no aparecen datos de pacientes.

Conflictos de interés: no hay.

\section{Referencias}

1. How P, Stelzner S, Branagan G, Bundy $\mathrm{K}$, Chandrakumaran K, Heald RJ, et al. Comparative quality of life in patients following abdominoperineal excision and low anterior resection for low rectal cancer. Dis Colon Rectum 2012;55:400-6.

2. Digennaro R, Tondo M, Cuccia
F, Giannini I, Pezzolla F, Rinaldi

$\mathrm{M}$, et al. Coloanal anastomosis or abdominoperineal resection for very low rectal cancer: what will benefit, the surgeon's pride or the patient's quality of life? Int J Colorectal Dis. 2013;28:94957.

3. Miles EW. A method of performing abdominoperineal excision for carcinoma of the rectum and of the terminal portion of the pelvic column. Lancet 1908;2:18123.

4. Sprangers MA, Taal BG, Aaronson NK, Te Velde A. Quality of life in colorectal cancer. Stoma vs nonstoma patients. Dis Colon Rectum 1995;38:361-9.

5. Schiessel R, Karner-Hanusch J, Herbst F, Teleky B, Wunderlich M. Intersphincteric 
resection for low rectal tumours. Br J Surg. 1994;81:1376-8.

6. Kim HS, Ko S, Oh NG. Long-term results of extended intersphincteric resection for very low rectal cancer: a retrospective study. BMC Surg. 2016;16: 21.

7. Martin ST, Heneghan HM, Winter DC. Systematic review of outcomes after intersphincteric resection for low rectal cancer. Br J Surg. 2012;99:603-12.

8. Akagi Y, Kinugasa T, Shirouzu K. Intersphincteric resection for very low rectal cancer: a systematic review. Surg Today 2013;43:838-47.

9. Saito N, Moriya Y, Shirouzu K, Maeda K, Mochizuki H, Koda K, et al. Intersphincteric resection in patients with very low rectal cancer: a review of the Japanese experience. Dis Colon Rectum 2006;49 (10 Suppl):S13-22.

10. Beppu N, Kimura H, Matsubara N, Tomita N, Yanagi H, Yamanaka N. Long-term functional outcomes of total mesorectal excision following chemoradiotherapy for lower rectal cancer: Stapled anastomosis versus intersphincteric resection. Dig Surg. 2016;33:33-42.

11. Klose J, Tarantino I, Kulu Y, Bruckner T, Trefz S, Schmidt T, et al. Sphincterpreserving surgery for low rectal cancer: Do we overshoot the mark? J Gastrointest Surg. 2017;21:885-91.

12. Jorge JM, Wexner SD. Etiology and management of fecal incontinence. Dis Colon Rectum. 1993;36:77-9.

13. Saito N, Ito M, Kobayashi A, Nishizawa Y, Kojima M, Nishizawa Y, et al. Longterm outcomes after intersphincteric resection for low-lying rectal cancer. Ann Surg Oncol. 2014;21:3608-15.

14. Emmertsen KJ, Laurberg S. Low anterior resection syndrome score: development and validation of a symptom-based scoring system for bowel dysfunction after low anterior resection for rectal cancer. Ann Surg. 2012;255:922-8.

15. Kirwan WO, Turnbull RB Jr, Fazio VW, Weakley FL. Pullthrough operation with delayed anastomosis for rectal cancer. $\mathrm{Br} \mathrm{J}$ Surg. 1978;65:695-8.

16. Luca F, Valvo $M$, Guerra-Cogorno $M$, Simo D, Blesa-Sierra E, Biffi R, et al. Functional results of robotic total intersphincteric resection with hand-sewn coloanal anastomosis. Eur J Surg Oncol.
2016;42:841-7.

17. Tsukamoto S, Kanemitsu Y, Shida D, Ochiai H, Mazaki J. Comparison of the clinical results of abdominoperineal intersphincteric resection and abdominoperineal resection for lower rectal cancer. Int J Colorectal Dis. 2017; 32:683-9.

18. Scala D, Niglio A, Pace U, Ruffolo F, Rega D, Delrio P. Laparoscopic intersphincteric resection: indications and results. Updates Surg. 2016; 68:85-91.

19. Kim CH, Lee SY, Kim HR, Kim YJ. Factors associated with oncologic outcomes following abdominoperineal or intersphincteric resection in patients treated with preoperative chemoradiotherapy: A propensity score analysis. Medicine 2015;94:e2060. Published online 2015 Nov 13. doi: [10.1097/ MD.0000000000002060]

20. Chi P, Huang SH, Lin HM, Lu XR, Huang Y, Jiang WZ, et al. Laparoscopic transabdominal approach partial intersphincteric resection for low rectal cancer: surgical feasibility and intermediate-term outcome. Ann Surg Oncol. 2015;22:944-51.

21. Pai VD, De Souza A, Patil P, Engineer $\mathrm{R}$, Arya S, Saklani A. Intersphincteric resection and hand-sewn coloanal anastomosis for low rectal cancer: Shortterm outcomes in the Indian setting. Indian J Gastroenterol. 2015;34:23-8.

22. Tekkis P, Tan E, Kontovounisios C, Kinross J, Georgiou C, Nicholls RJ, et al. Hand-sewn coloanal anastomosis for low rectal cancer: technique and long-term outcome. Colorectal Dis. 2015;17:106270.

23. Koyama M, Murata A, Sakamoto Y, Morohashi H, Takahashi S, Yoshida E, et al. Long-term clinical and functional results of intersphincteric resection for lower rectal cancer. Ann Surg Oncol. 2014;21 Suppl 3:S422-8.

24. Zhang B, Zhao K, Liu Q, Yin S, Zhuo $\mathrm{G}$, Zhao Y, et al. Clinical and functional results of laparoscopic intersphincteric resection for ultralow rectal cancer: is there a distinction between the three types of hand-sewn colo-anal anastomosis? Int J Colorectal Dis. 2017;32:587-90.

25. Yokota M, Ito M, Nishizawa Y, Kobayashi A, Saito N. The Impact of Anastomotic
Leakage on Anal Function Following Intersphincteric Resection. World J Surg. 2017;41:2168-77.

26. Yoo BE, Cho JS, Shin JW, Lee DW, Kwak JM, Kim J, et al. Robotic versus laparoscopic intersphincteric resection for low rectal cancer: comparison of the operative, oncological, and functional outcomes. Ann Surg Oncol. 2015;22: 1219-25.

27. Lelong B, de Chaisemartin C, Meillat $\mathrm{H}$, Cournier S, Boher JM, Genre D, et al; French Research Group of Rectal Cancer Surgery (GRECCAR). A multicentre randomised controlled trial to evaluate the efficacy, morbidity and functional outcome of endoscopic transanal proctectomy versus laparoscopic proctectomy for low-lying rectal cancer (ETAP-GRECCAR 11 TRIAL): rationale and design. BMC Cancer 2017; 17:253. Published online 2017 Apr 11. doi: [10.1186/s12885-017-3200-1].

28. Yamada K, Ogata S, Saiki Y, Fukunaga M, Tsuji Y, Takano M. Long-term results of intersphincteric resection for low rectal cancer. Dis Colon Rectum 2009;52:106571.

29. Han JG, Wei GH, Gao ZG, Zheng Y, Wang ZJ. Intersphincteric resection with direct coloanal anastomosis for ultralow rectal cancer: the experience of People's Republic of China. Dis Colon Rectum 2009;52:950-7.

30. Chamlou R, Parc Y, Simon T, Bennis M, Dehni N, Parc R, et al. Long-term results of intersphincteric resection for low rectal cancer. Ann Surg. 2007;246:916-21.

31. Horisberger K, Rothenhoefer S, Kripp M, Hofheinz RD, Post S, Kienle P. Impaired continence function five years after intensified chemoradiation in patients with locally advanced rectal cancer. Eur J Surg Oncol. 2014;40:227-33.

32. Cong JC, Chen CS, Ma MX, Xia ZX, Liu DS, Zhang FY. Laparoscopic intersphincteric resection for low rectal cancer: comparison of stapled and manual coloanal anastomosis. Colorectal Dis. 2014; 16:353-8.

33. Tokoro T, Okuno K, Hida J, Ueda K, Yoshifuji T, Daito K, et al. Analysis of the clinical factors associated with anal function after intersphincteric resection for very low rectal cancer. World J Surg Oncol. 2013;11:24. 